\section{LA PARTICIPACIÓN DEL PÚBLICO EN EL COSTE DE LA OFERTA CULTURAL: ARGUMENTOS ÉTICOS PARA EL DEBATE}

\author{
Rafael Cejudo Córdoba \\ Universidad de Córdoba \\ rafael.cejudo@uco.es \\ ORCID iD: http://orcid.org/0000-0003-1184-6258
}

\section{PARTICIPATION OF THE PUBLIC IN FUNDING OF THE CULTURAL OFFER: ETHICAL ARGUMENTS FOR DEBATE}

Cómo citar este artículo/Citation: Cejudo Córdoba, R. (2017). La participación del público en el coste de la oferta cultural: argumentos éticos para el debate. Arbor, 193 (784): a387. doi: http://dx.doi.org/10.3989/arbor.2017.784n2009
Copyright: (C) 2017 CSIC. Este es un artículo de acceso abierto distribuido bajo los términos de la licencia Creative Commons Attribution (CC BY) España 3.0.
RESUMEN: El artículo revisa los argumentos a favor y en contra de que el público financie directa y voluntariamente la actividad cultural. Se parte de que los mecanismos de financiación elegidos por las políticas culturales tienen una dimensión ética y no solo una finalidad instrumental. Utilizando el enfoque comparativo propuesto por $\mathrm{A}$. Sen, se investiga si el micro-mecenazgo (crowdfunding) y otras formas de inversión por parte de los consumidores culturales constituyen formas de mecenazgo ciudadano valiosas desde un punto de vista ético. A pesar de que las políticas culturales a favor del micro-mecenazgo pueden ser acusadas de paternalistas o de mercantilizar la vida cultural, se defiende que el mecenazgo ciudadano es una innovación social que fomenta la responsabilidad social de los consumidores culturales. En tal sentido las políticas culturales deben tener en cuenta que el comportamiento privado de consumo e inversión también es una forma de participación en la vida social.

PALABRAS CLAVE: Mecenazgo ciudadano; crowdfunding; consumo cultural; innovación social; A. Sen.
ABSTRACT: This paper reviews arguments for and against voluntary and direct participation of the public in the funding of cultural activities. The starting point is that financing schemes implemented by cultural policies bear ethical implications aside from their instrumental aims. Using A. Sen's comparative approach, it is discussed whether crowdfunding and other investment instruments for cultural consumers are forms of citizen patronage worthwhile from an ethical point of view. In spite of accusations of paternalism and commoditization of the cultural life, citizen patronage is argued to be a social innovation that encourages social responsibility of cultural consumers. Accordingly, cultural policies should take into account that private behaviors concerning consumption and investment are also ways of getting involved in social life.

KEYWORDS: Citizen patronage; crowdfunding; cultural consumption; social innovation; A. Sen. 


\section{INTRODUCCIÓN}

En los años ochenta del pasado siglo, las políticas "neoliberales" en Gran Bretaña y otros lugares incluyeron una drástica reducción de la financiación pública de la actividad cultural, y la exigencia (a la fuerza ahorcan) de una participación del público mucho mayor para mantener una infraestructura y una actividad cultural siquiera parecidas (Collini, 1992). Incluso en Francia, donde el Estado Cultural (Fumaroli, 1991) es antiguo y fuerte, la legislación favorece desde 2003 las donaciones de particulares y empresas mediante la Loi relative au mécénat, aux associations et aux fondations. La reciente crisis económica ha reforzado este cambio de acento en la financiación de la Cultura ${ }^{1}$. En consecuencia, gobiernos tanto de un signo como de otro, tanto a nivel estatal como autonómico, han promovido un cierto cambio en la forma de financiar la actividad cultural².

Salvo en el caso de la Ley Foral 8/2014, de 16 de mayo, reguladora del mecenazgo cultural y de sus incentivos fiscales en la Comunidad Foral de Navarra, los demás cambios legislativos no han llegado a materializarse. De todas formas, la legitimidad de una mayor participación privada en la financiación de la Cultura se ha convertido ya en ortodoxia. Tal como advierten Bastien, Dubois, Freyermuth y Matz (2012), la reducción de los presupuestos públicos culturales se percibe como un dato básico e incuestionable. Simultáneamente, se asume que será más eficiente este nuevo e inevitable marco fiscal. Seguramente un cambio del ciclo económico traerá mejores perspectivas de gasto público, y con ello una menor urgencia de encontrar financiación privada. De todas formas, a la coyuntura económica hay que añadir otro factor mucho más duradero como el efecto de las redes de comunicación social tanto en la actividad económica como en la cultural (Benkler, 2006; Castells, 2000).

Las tecnologías de la información y la comunicación han permitido maneras inéditas de cooperación económica y social, y también formas nuevas de producir y acceder a la Cultura. Si no hace mucho la alternativa al Estado debían ser "mecenas reales o magnates intrépidos" (Feinberg, 1994, p. 101), hoy también los individuos particulares pueden realizar una financiación significativa de la actividad cultural (mecenazgo ciudadano) mediante las plataformas de crowdfunding o micro-mecenazgo. En general, por tanto, los consumidores culturales pueden participar en el coste de la oferta cultural pagando un precio por bienes culturales antes subvencionados, o también aportando capital a las empresas y pro- yectos que producen esos bienes, con o sin interés lucrativo por su parte. Hay un consenso generalizado sobre que lo más urgente en las nuevas normativas sobre financiación de la Cultura es lo relativo al crowdfunding (Ponce Velasco, 2013; Raussell Köster, Montagut Marqués y Minyana Beltran, 2013; Sajardo Moreno y Ribeiro Coutinho, 2014). Fuera de la academia, el tono es muchas veces encomiástico. Por ejemplo Y. Stricker, fundador de la plataforma de crowdfunding Kickstarter declara en su web: "En Kickstarter, los artistas e innovadores establecen sus propias reglas para sus creaciones y todas las ideas tienen la misma posibilidad de ser realizadas - ¡todo el mundo participa de forma igualitaria en la formación del futuro de la Cultura!". Y Brian Meece, de la plataforma RocketHub dice que "Beethoven + redes sociales = crowdfunding para la creación moderna. Bienvenidos al renacimiento del patronazgo" (Lawton y Marom, 2010, p. 3). No es la finalidad de este artículo describir las distintas modalidades de micro-mecenazago (Alemany y Bultó, 2014; Gutiérrez-Rubi y Freire, 2012; Rubio Arostegui, Rius Ulldemolins, y Martínez Illa, 2014) sino debatir si este último, junto con esas otras modalidades de financiación privada de la oferta cultural, puede defenderse por motivos éticos. Dicho de otra manera, si más allá de su jubilosa acogida por promotores y académicos, el micro-mecenazgo contribuye a la democracia cultural haciendo justicia a la denominación de "mecenazgo ciudadano".

El resto del artículo se estructura del modo siguiente: la sección segunda establece el marco desde el que se abordará la valoración del mecenazgo ciudadano. Además de precisar cómo se entenderá esa noción, se introduce también el concepto más amplio de sistema de financiación de la Cultura (SFC), defendiéndose su relevancia ética además de económica. Así mismo se explica que la valoración del mecenazgo ciudadano como modalidad de financiación se realiza mediante un enfoque comparativo. La sección tercera se ocupa tanto de argumentos contrarios al mecenazgo ciudadano, como de razones en contra de utilizar el SFC para mejorar la calidad ética de la vida social. La cuarta sección expone distintos argumentos a favor del mecenazgo ciudadano basados justamente en su impacto positivo sobre la ciudadanía. Puesto que el artículo no pretende realizar ese ejercicio escolástico de reunir argumentos, réplicas y contra-réplicas, la sección final señala algunos resultados obtenidos, las limitaciones del enfoque utilizado y las implicaciones para el debate público sobre la financiación de la Cultura. 


\section{PRECISIONES METODOLÓGICAS}

La cuestión del micro-mecenazgo va unida a la del marco institucional que lo acoja. La legislación al respecto podrá ser un impulsor o un freno, favoreciendo más o menos tanto las plataformas de crowdfunding (al fin y al cabo, nuevas empresas de intermediación financiera), como a los inversores clientes de esas empresas (mediante desgravaciones fiscales, por ejemplo). Junto con las demás medidas para favorecer la participación de los ciudadanos en el coste de la oferta cultural, constituyen un sistema de financiación de la Cultura (SFC) alternativo o, de manera más realista, complementario al SFC donde el Estado financia la actividad cultural vía impuestos. Aparte de las cuestiones, fundamentales desde luego, de cuáles sean las causas de un SFC y de cuán eficiente sea (Albi Ibáñez, 2003; De la Torre Sotoca, 2013), consideraré que la forma de pagar por un bien, y más concretamente de financiar el tipo especial de bienes propios del ámbito de la Cultura, es una institución social y política con relevancia ética. Para abordar esta dimensión utilizaré una forma de razonar común en ética aplicada, cual es reflexionar sobre la finalidad adecuada de una institución. No me refiero a qué finalidad deben tener los fondos recaudados mediante el sistema de financiación (y que, en términos generales, podrían ir desde la redistribución de la riqueza hasta sufragar un Estado mínimo); la cuestión que abordaré es la finalidad del propio diseño institucional.

Usualmente esa cuestión del diseño institucional no se considera filosófica o ética, sino un asunto empírico que deben decidir políticos y gestores ayudados, en todo caso, por científicos sociales. La filosofía se ocuparía solo de los valores y finalidades generales que se deben perseguir (tal como "las instituciones económicas deben promover la igualdad", p. ej., o "las políticas culturales deben respetar la libertad de expresión"). La cuestión moral sería qué debe hacerse, y la institucional quién y cómo debería hacerlo. Como hacen otros autores (R. Claassen, 2011; Keat, 2011), parto de cuestionar esta división del trabajo por la cual solo a las ciencias sociales les corresponde determinar ventajas e inconvenientes de los diseños institucionales concretos (tales como qué tipo de impuestos son más igualitarios o qué tipo de legislación sobre derechos de autor protege más la libertad de expresión). Estas preguntas institucionales son también preguntas morales de un tipo especial.

Son finalidades necesarias de cualquier sistema de financiación, también del SFC, la eficiencia, el control del equilibrio presupuestario y la equidad en la re- caudación. Teniendo en cuenta lo último, una crítica importante de un SFC basado en contribuciones privadas voluntarias ( $p$. ej. mediante micro-mecenazgo y ventas a precio de mercado de los bienes culturales), es que el acceso a la Cultura dependería de la renta disponible y de la buena voluntad de los mecenas. No se puede pagar cuando no se tiene, de modo que la privatización de la financiación atentaría contra el derecho a la Cultura, reconocido p. ej. en el art. 27 de la Declaración Universal de los Derechos Humanos o en el art. 9 de la Constitución Española. Para sortear esta objeción haré una observación y una hipótesis. La primera es que "los almuerzos gratis no existen", en la frase popularizada por Milton Friedman. Dicho de otra manera, la financiación pública de los bienes culturales no es gratuita puesto que la sufragan lógicamente individuos y empresas a través de sus impuestos. Por otra parte, voy a considerar por hipótesis que el gasto cultural de todas las administraciones del Estado sea el mismo en un SFC1 donde toda la financiación es con cargo a los presupuestos del Ministerio de Cultura, consejerías y concejalías correspondientes, y otro SFC2 donde el Estado gasta lo mismo en Cultura pero con cargo directo a presupuestos solo para mantener ciertos servicios culturales básicos, siendo el resto del gasto mediante incentivos fiscales a la producción y al consumo cultural. Aunque sea una cuestión clave determinar qué bienes culturales tienen que sufragarse directamente por el Estado incluso en SFC2 para garantizar así el derecho a la Cultura, dicha cuestión no es relevante para mi argumentación. Quede claro en todo caso que lo más realista será siempre alguna combinación de SFC1 y SFC2. La idea de un SFC basado exclusivamente en contribuciones privadas y voluntarias solo debe entenderse como una hipótesis que cumpla su función en el curso de la argumentación. Qué SFC concreto sea mejor dependerá en parte de consideraciones empíricas imposibles de controlar plenamente. Por esa razón, qué tipo de SFC es más adecuado no puede determinarse a priori. Mi objetivo es argumentar sobre las ventajas éticas de sistemas de financiación más cercanos a SFC1 que a SFC2, esto es, sistemas de financiación donde los ciudadanos participen significativamente más que en la actualidad en el coste de los bienes culturales, tanto desde el lado de la oferta (medidas de apoyo al crowdfunding) como de la demanda (desgravaciones al consumo cultural). Denominaré mecenazgo ciudadano a estos tipos de SFC en que los ciudadanos contribuyen significativamente con su propia renta disponible a sufragar la oferta cultural de su sociedad. 
Valorar el mecenazgo ciudadano es un caso particular del problema de ética aplicada consistente en estimar la calidad ética de sistemas o instituciones alternativas. En este punto, y como A. Sen, considero inadecuado lo que él llama institucionalismo trascendental, consistente en imaginar la institución perfecta en lugar de centrarse en comparaciones relativas entre las ya existentes, despreocupándose así de las sociedades reales en que las instituciones emergen (Sen, 2009, pp. 5-6). En su lugar utilizaré lo que Sen llama enfoque comparativo, según el cual la calidad ética de un sistema $X$ siempre es relativa a otro vecino en el espacio o en el tiempo. Tal como dice Echeverría (2008, p. 611), "aunque no existe una unidad que mida el bienestar social o la calidad de vida, esos valores pueden ser medidos en escala comparativa". Se trata por lo tanto de usar la metodología de las ordenaciones parciales. Una ordenación es completa cuando para cualquier par de alternativas $x$ e $y$, o bien $x$ es al menos tan buena como $y$, o bien $y$ es al menos tan buena como $x$. Cuando la ordenación es parcial, no puede decidirse en todos los casos qué alternativa es mejor, pero sí puede tomarse tal decisión en los casos comparables, lo que puede ser suficiente desde un punto de vista práctico (Sen, 1970). En suma, no se trata de determinar cuál es el mejor de los SFC posibles sino, por ejemplo, si el apoyo al mecenazgo cultural es una mejora coeteris paribus. Resulta obvio que el enfoque comparativo no libera de encontrar criterios de comparación, antes al contrario.

Por otro lado, esos criterios de comparación deberían tener en cuenta a todos los agentes que tienen algún interés en la Cultura. Tales grupos de interés abarcan desde la sociedad en general hasta el propio Estado, pasando por los consumidores y los productores culturales (ya sean autores o gestores culturales). Las mejoras y perjuicios éticos para cada grupo son distintos. Por ejemplo, para los autores diferentes SFC implican otros tantos niveles de libertad creativa, y para la sociedad en general una vida cultural más o menos rica. Este artículo se limita a las repercusiones morales del SFC sobre los consumidores. La situación de partida es una gran presencia pública en la financiación de la Cultura. En el caso español, el gasto estatal en Cultura ascendió a poco más 4.000 millones de euros en 2013 (un 3,3\% del presupuesto total del Estado), mientras que los consumidores se gastaron en libros, espectáculos escénicos y museos 2.418 millones de euros. El capítulo de gasto estatal más importante es el del patrimonio histórico-artístico, que ascendió a 351 millones de euros, mientras que los consumidores solo se gastaron en ello 127 millones
(Anuario de Estadísticas Culturales 2015, pp. 87-117). De manera similar, un estudio de la Fundación Contemporánea indica que los consumidores solo financian un $31,8 \%$ de la oferta cultural, y en más de la mitad de los casos su contribución baja a menos del $20 \%$ (Observatorio de la Cultura. Primer semestre de 2013. Análisis de resultados). El Estado, por tanto, financia el $80 \%$ de los bienes culturales en más de la mitad de los casos. Estas decisiones de financiación implican que determinados bienes culturales tengan más o menos posibilidades de llegar finalmente al público. Sin necesidad de que haya censura, el Estado ejerce un control importante de la oferta cultural, dado que además esta no puede financiarse fácilmente mediante los mecanismos de mercado habituales en otros sectores (Baumol y Bowen, 1966; Ginsburgh y Throsby, 2013). En consecuencia, un SFC será tanto mejor cuanto más contribuya a la autonomía de criterio y a la libertad de elección de los consumidores finales de bienes y servicios culturales, y en este sentido será tanto mejor cuanto más haga posible la soberanía del consumidor cultural frente a las imposiciones de la industria cultural o del propio Estado.

\section{ARGUMENTOS CONTRA EL MECENAZGO CIUDADANO}

Siguiendo el mencionado enfoque comparativo, no se pretende aquí aportar una lista cerrada de argumentos, sino ofrecer elementos de juicio con los que contrastar las ventajas éticas del mecenazgo ciudadano que se expondrán en la siguiente sección. Comenzaré indicando cuatro motivos por los que la participación directa y voluntaria del público en la financiación de la Cultura debería tomarse con suma cautela. En todos ellos el problema de fondo sería la difícil compatibilidad entre la actividad cultural y la actividad de mercado. Finalmente me referiré a la argumentación de J. Rawls a favor de la neutralidad de las instituciones, y por tanto a la acusación de que el Estado utilizaría la Cultura de modo paternalista si estableciera el marco institucional para su financiación con objetivos morales tales como educar o empoderar a los ciudadanos.

En primer lugar, si consumo y Cultura fueran una síntesis imposible, entonces la soberanía del consumidor cultural sería absurda $a b$ initio. Adorno, Horkheimer o Arendt pensaban justamente que la dimensión liberadora de la Cultura pasa porque esta no se consume (Adorno, 1977; Arendt, 1958; Horkheimer y Adorno, 1984). El mecenazgo ciudadano parte de lo contrario, de que sí es posible reconciliar consumo y Cultura, aunque ello implique en- 
tender ambos de manera distinta a la de los autores citados (Rodríguez Ferrándiz, 2011). Por otro lado, se puede también considerar que, aunque la soberanía del consumidor cultural es posible, no puede ser democrática, y que por tanto no vale la pena perseguirla cuando el acceso a la Cultura está protegido incluso constitucionalmente. El problema ahora es que el mecanismo de mercado no asigna los bienes $y$ servicios según criterios de necesidad, de modo que solo los consumidores más pudientes podrían participar significativamente en el mecenazgo ciudadano. Mientras tanto, los demás ciudadanos quedarían culturalmente desatendidos. Si la soberanía del consumidor significa que "los consumidores votan con sus dólares" (Persky, 1993, p. 185), está claro que eso no es admisible en la esfera pública pues solo los más ricos podrían votar. En consecuencia, por criterios de justicia distributiva, es necesario renunciar a la soberanía del consumidor cultural a favor de la financiación pública de los bienes culturales.

Un tercer argumento se basa en los límites que debería tener el mercado. Incluso aunque todos los consumidores tuvieran la misma renta disponible, ahora se trata de que el SFC no debe utilizar criterios mercantiles. El mecenazgo ciudadano, por más que en muchos casos tenga una motivación altruista, introduce también criterios monetarios que distorsionan las reglas internas de la práctica cultural. Siguiendo a Maclntyre, Keat (2000) argumenta que la producción cultural es una práctica con unos criterios de éxito propios y distintos de la satisfacción de las preferencias de los consumidores (una obra de arte, por ejemplo, no es necesariamente peor porque no encuentre comprador). Más aún, en la relación que el consumidor cultural traba con los bienes culturales, sus preferencias no siempre son un dato pre-establecido, sino que pueden formar parte de esa relación. Dicho de otra manera, esas preferencias se conforman en el seno de unas prácticas en las que los consumidores se involucran asumiendo el papel de público (espectador, lector, etc.), y en conformidad con tal rol asumen voluntariamente una actitud de espera, aceptación o compenetración con los productos culturales. En consecuencia, las preferencias del consumidor no anteceden al objeto cultural consumido ni pueden por tanto determinar la producción cultural. Al contrario, tales preferencias son conformadas por esa práctica cultural en la que acepta participar como consumidor no soberano, puesto que asume sus criterios de excelencia internos, criterios que son los verdaderos "soberanos" y que solo el productor (no el consumidor) acierta en desentrañar.
La anterior descripción de la experiencia cultural no siempre se corresponde con la realidad porque hoy las propias prácticas culturales conceden al público consumidor un papel destacado (Bauman, 1997). Aun así, para muchos consumidores no expertos "el valor de ciertas prácticas culturales podría verse como residiendo parcialmente en su contribución a las experiencias educativas involucradas en adquirir y ejercitar la pericia de hacer genuinos juicios valorativos" según dice Keat (2000, p. 32). Así pues, el carácter específico de la Cultura excluye al consumidor como árbitro supremo del valor de los bienes y servicios culturales. En conclusión, el consumidor cultural no puede ser consumidor soberano precisamente por ser cultural. Y en consecuencia, el productor cultural está legitimado para rechazar una relación de mercado en la que será financiado solo si satisface las preferencias de los demandantes, puesto que las reglas internas de la práctica cultural le enfrentan con exigencias distintas. Incluso se podría aducir que el mecenas clásico aceptaba la autoridad del artista en muchos casos, sometiendo su juicio al prestigio de este último. En cambio, precisamente porque la relación con los micro-mecenas es mucho más igualitaria y democrática, las reglas de la práctica cultural se adulteran al conceder una importancia excesiva a los consumidores.

Finalmente, los límites que debería tener el mercado proporcionan un cuarto argumento en contra de la viabilidad del mecenazgo ciudadano. Tal como indica Keat (2011, p. 68), muchas personas que defienden la emisión de programas sobre arte en las cadenas públicas de televisión nunca los ven, y si tuvieran que pagar por su emisión tampoco lo harían. En otros ejemplos de la vida cultural pasa algo semejante (la gente puede estar orgullosa de la orquesta local, pero jamás ir a la sala de conciertos; o puede querer que se enseñe a Cervantes en las escuelas, pero no estar dispuesta a gastarse un euro en la investigación de la literatura cervantina). $Y$ más en general, es perfectamente razonable valorar $X$ y no estar dispuesto a pagar por $X$, simplemente porque las preferencias de compra no expresan todas las preferencias del individuo (p. ej. porque un aumento de mi renta disponible modificará mi comportamiento de consumo). En consecuencia, resulta útil distinguir el comportamiento como consumidor del comportamiento como ciudadano: como consumidor no estoy dispuesto a gastar en Cultura ni un euro más de mi presupuesto, pero como ciudadano sí estoy dispuesto a que se gaste un euro más del presupuesto público en Cultura. Y dado que los bienes culturales no son bienes públicos que beneficien a todos por igual, lo más adecuado es que la financiación 
se decida por el partido más votado en lugar de por las compras o inversiones de los consumidores (Anomaly, 2015; R. Claassen, 2013).

Otro tipo de críticas se dirigen no al mecenazgo ciudadano como tal, sino al más amplio propósito político de implantar un SFC cuya finalidad sea que los consumidores culturales se hagan mejores ciudadanos (más informados, más críticos, más responsables), y en ese sentido se mejore la calidad moral de la vida social mediante la política cultural (mediante su financiación concretamente). Si esta mejora moral se limita al rol de ciudadano y, más específicamente, a la actuación ciudadana en el campo cultural, entonces el inconveniente de que tal SFC sería inadmisiblemente paternalista solo podría ser invocado por una parte de la filosofía política liberal contemporánea, eso sí, por una muy influyente. La argumentación sería de este tipo: dado que los bienes y servicios culturales responden a preferencias subjetivas (ya sean estas de tipo estético o puramente hedonista es indiferente), tales bienes y servicios pertenecen a una esfera privada donde el Estado no debe entrometerse. Como en tantos otros casos, el planteamiento de J. Rawls también aquí es paradigmático, ya sea en relación con el asunto concreto de las políticas culturales (y a fortiori de su financiación pública), como en el más general de la neutralidad que deben tener las instituciones políticas (lo que incluiría el SFC). En ambos casos la neutralidad del Estado respecto de la esfera de valores privados excluiría utilizar el SFC como un instrumento a favor de la emancipación y el empoderamiento ciudadano. La tesis rawlsiana sobre la financiación de la Cultura es que "no tendría más justificación usar el aparato del Estado para que algunos ciudadanos paguen a la fuerza beneficios indeseados, que la que habría en obligarles a reembolsar a otros sus propios gastos privados" (Rawls, 1971, p. 283). Tales "beneficios indeseados" pueden ser los bienes y servicios culturales financiados por el Estado puesto que responderían a preferencias meramente subjetivas. Dicho de otra manera: pagar por la Cultura de otros es paternalista puesto que el Estado debe ser neutral respecto de las concepciones alternativas sobre la buena vida, y es asunto de cada cual elegir qué bienes culturales son los que le enriquecen. La cultura política estadounidense cuadra con esta línea liberal de argumentación pues, como dice Cunnane (1999, p. 1445) a propósito del pleito National Endowment for the Arts v. Finley: "el gobierno de Estados Unidos no está obligado a financiar el arte. Ni la Primera Enmienda ni ninguna otra cláusula constitucional establecen tal obligación".

\section{EL MECENAZGO CIUDADANO COMO MEJORA MORAL}

Tras haber repasado argumentos éticos contrarios a la participación directa del público en la financiación de la Cultura, en esta sección se exponen argumentos más poderosos a favor de la misma. Para comenzar, téngase en cuenta que son cuestiones distintas si, (a), un SFC puede mejorar moralmente a los consumidores culturales haciéndolos de algún modo mejores ciudadanos y si, (b), dado que ese sea el caso, el Estado debería por ello implantar tal SFC. Me ocuparé primero de argumentar a favor de (b) antes de exponer varias formas en que el micro-mecenazgo y las deducciones fiscales al consumo cultural pueden hacer mejores ciudadanos. Como he explicado al final de la sección anterior mediante la argumentación de Rawls, la cuestión (b) debe hacer frente a la objeción del paternalismo ${ }^{3}$. En todo caso, a favor de (b) o de por qué deberíamos preferir la implantación de SFC (y de sistemas de financiación en general) que hagan mejores ciudadanos, una primera respuesta es que dados dos SFC igualmente eficientes y equitativos, si uno de ellos además mejora moralmente a los consumidores culturales, entonces es preferible al otro. Pero esto no pasa de ser una defensa ocasional de (b). Una vez que se admite que el diseño institucional, y concretamente el de la fiscalidad de la actividad cultural, tiene una dimensión ético-política, la respuesta a (b) depende de cuáles han de ser las funciones del Estado y de la política en general.

Solo en la reciente filosofía política liberal (señaladamente Rawls), se defiende que el Estado debe ser absolutamente neutral y no inclinarse por ninguna concepción del bien humano en particular. Pero frente a esta tesis, y por más que en el ámbito de la reflexión pura apenas haya certezas, cuando la conversación desciende a temas concretos sí surgen muchas certidumbres sobre qué cosas mejoran la vida humana y cuáles no (Galston, 1991). En suma, todas las formas de asociación política tienen que justificarse con alguna referencia a qué sea bueno para los que integran la asociación. Por eso, en el terreno de los hechos, tanto los gobiernos democráticos de cualquier signo como las instituciones internacionales dan por sentado bienes humanos como la salud, la educación o la seguridad personal. Y cuando nos apartamos algo de la ortodoxia ralwsiana, hallamos varias propuestas que defienden una concepción no paternalista de los bienes humanos que deben ser objetivos políticos.

La concepción de Rawls puede calificarse como neutralismo cultural, o mejor perfeccionismo liberal 
débil, puesto que Rawls defiende que el Estado debe favorecer una educación moral de los ciudadanos y que el objetivo de la misma es que los ciudadanos sean más autónomos (Rawls, 1971, p. 516). Sin embargo, esa educación se limita a la aceptación voluntaria de los Principios de Justicia y de sus consecuencias, tal como hacen los miembros de la situación original. Un paso más allá sería lo que denominaré perfeccionismo liberal fuerte en cuestiones culturales, defendido por Raz, Dworkin, Nussbaum o Sen. Según estos autores, las personas tienen capacidades en cuyo desarrollo radica el bien humano, y en consecuencia las instituciones políticas deberían proporcionar un rango de opciones realista y suficientemente amplio que permita elegir a los individuos cuáles capacidades quieren desarrollar (Dworkin, 1985; Keat, 2011; Nussbaum, 1990; Raz, 1986; Sen, 1999). Aquí el Estado no fomenta ningún modelo de buena vida, pero proporciona el marco para que los distintos modelos puedan llevarse a la práctica. Un paso más allá sería el progresismo liberal en cuestiones culturales, donde la función de las políticas no es asegurar algún SFC que permita a los ciudadanos desarrollar sus capacidades, sino determinar qué SFC desarrolla esas capacidades. En el perfeccionismo liberal fuerte, las capacidades son para lograr los fines de unas prácticas previamente dadas (culturales, deportivas, personales) o que son conformadas por decisiones individuales. En cambio, el progresismo liberal persigue tanto unas prácticas, como una forma de financiar esas prácticas, que mejoren a los ciudadanos en el sentido de desarrollar su capacidad para imaginar y perseguir fines (Casado da Rocha, 2013; Siurana, 2009).

El planteamiento de J. S. Mill es ilustrativo del progresismo liberal cultural. Según Mill el objetivo más importante de un gobierno es promover la virtud y la inteligencia de la ciudadanía, lo cual no es muy distinto de maximizar el bienestar social. De aquí se sigue que las políticas y acciones de gobierno deben juzgarse según dos criterios ético-políticos: uno es hasta qué punto son capaces de aprovechar las capacidades de los gobernados para lograr bienestar social; el otro criterio es el grado en que tienden a incrementar el total de buenas cualidades de los gobernados, tanto en sentido individual como colectivo (J. S. Mill, 1977a, pp. 390-391). Por tanto, deberíamos preferir un SFC que incremente las buenas cualidades de los ciudadanos, o que aproveche esas buenas cualidades para una vida cultural que redunde en mayor bienestar social, en lugar de un SFC que no lo haga.
La cuestión (a) indicada al principio de esta sección consistía en si el mecenazgo ciudadano es un SFC que implica una mejora moral, en si el mecenazgo ciudadano incrementa las buenas cualidades del público y por tanto debería preferirse coeteris paribus. La respuesta puede buscarse desde el lado de los productores culturales o del propio Estado, y no solo limitarse a los consumidores. En cualquier caso, uso el término "mejora" deliberadamente, remitiéndome al empleo que J. S. Mill (1977b) hacía de ese término (improvement) en el sentido de ser una medida intermedia, un pequeño paso en la buena dirección, incluso si inicial o parcialmente acarrea incertidumbres o inconvenientes. En consecuencia, las mejoras nunca son logros definitivos ni absolutos, sino relativos y graduales. A pesar de ello, son suficientes para justificar un juicio ético a favor de determinada alternativa de acuerdo con el enfoque comparativo. También por ello mismo, no es ningún inconveniente que la lista de mejoras quede abierta, pues la finalidad de la ordenación parcial no es determinar el SFC perfecto sino colaborar en el diseño institucional. Así pues, el mecenazgo ciudadano conlleva al menos las mejoras siguientes: (1) es una innovación social que (2) fomenta la responsabilidad social de los consumidores culturales, (3) apoyando la soberanía del consumidor cultural.

Comenzaré por la primera de estas mejoras. Ciertas modalidades de mecenazgo ciudadano, como el crowdfunding o los fondos de inversión cultural, son innovaciones "incrementales" (Schumpeter, 1934) en cuanto mejoras que encajan en un proceso de cambio que enlaza con la cuestación y la suscripción popular. Schumpeter propuso que la innovación podía ser un nuevo producto, un cambio cualitativo en un producto existente, un nuevo sistema productivo, un nuevo mercado, nuevos factores productivos o incluso una nueva organización industrial. Los tipos de financiación indicados encajan en varias de estas categorías: son nuevas formas de financiación (los fondos de inversión cultural), o cambios cualitativos en algo que ya existía (el crowdfunding), que permitirán por otro lado el acceso a "nuevos mercados", esto es, la participación de más públicos en el coste de la oferta. Por otro lado, se trata de innovaciones sociales y no meramente económicas. La innovación social tiene que referirse a objetivos y valores sociales como la felicidad y el bienestar, la calidad de vida, la inclusión social, la solidaridad, la participación ciudadana, la calidad medioambiental, la atención sanitaria, la eficiencia de los servicios públicos o el nivel educativo de una sociedad (Bornstein y Davis, 2010; Echeverría, 2008; Fowler, 2000; Mul- 
gan, 2006; Murray, Caulier-Grice y Mulgan, 2010; Nicholls y Murdock, 2012). El mecenazgo ciudadano encajaría en el tipo innovación cultural, puesto que se proponen nuevas infraestructuras culturales (lo que incluye los dispositivos tecnológicos requeridas por el nuevo SFC), o nuevas formas de participación en la vida cultural (una mayor implicación del consumidor cultural en el producto, pues el mecenazgo ciudadano supone participar también en la fase productiva del mismo). Y más allá de estos aspectos más o menos formales, la expectativa creada por el mecenazgo ciudadano en cuanto innovación cultural va más allá: los consumidores culturales se convertirían en usuarios y agentes expertos al ser la financiación mediante micro-mecenazgo descentralizada y no jerarquizada.

En segundo lugar, un SFC que apoye el mecenazgo ciudadano fomenta la responsabilidad social de los consumidores culturales. La noción de "responsabilidad social" procede de la ética de la empresa. La responsabilidad social de las empresas (RSE) puede definirse como "la integración voluntaria, por parte de las empresas, de las preocupaciones sociales y medioambientales en sus operaciones comerciales y sus relaciones con sus interlocutores" (Libro Verde. Fomentar un marco europeo para la responsabilidad social de las empresas, 2001). Las medidas de RSE son iniciativas voluntarias (y en tal sentido éticas) que ocasionan beneficios sociales. La responsabilidad social de los consumidores indica el deber moral que estos tienen de "evitar daños sociales e incluso de actuar proactivamente en pos del beneficio social, lo que podría incluir las tres facetas del comportamiento del consumidor: obtener, usar y desechar" (Vitell, 2015, p. 768). Un SFC que apoye el mecenazgo ciudadano supone trasladar a los ciudadanos la responsabilidad de obtener aquellos bienes culturales que más valoren individualmente o que consideren que deben ser accesibles al público. En general, la responsabilidad social del consumidor justifica obligaciones de los ciudadanos para con la sociedad que no se limitan al ámbito político (como defiende el liberalismo y el republicanismo), sin que tampoco sea necesario recurrir a una legitimación comunitarista de las políticas públicas.

En tercer lugar, el mecenazgo ciudadano apoya la soberanía del consumidor cultural. Como descripción de un hecho, la soberanía del consumidor apenas existe en un mundo dominado por grandes corporaciones económicas y financieras. Pero cuando en 1936 W. H. Hutt propuso esta noción, lo hizo para defender el poder de los ciudadanos frente al autoritarismo político de entreguerras (Persky, 1993). En una economía de mercado, los consumidores son soberanos porque no han sido privados del poder para demandar o no demandar recursos valiosos y escasos. En la concepción de Hutt, la producción es un instrumento para la consecución de fines, mientras que el consumo trata esencialmente sobre cuáles son esos fines. Así pues, en una sociedad de mercado cada ciudadano es siervo de la sociedad en cuanto productor, pero dirige las decisiones de los productores en cuanto consumidor (Hutt, 1990). El propio Hutt reconocía que las desigualdades en poder de compra hacían que la soberanía del consumidor fuera difícilmente viable sin una democracia de consumidores. En cualquier caso, en los SFC donde el Estado es quien paga la mayor parte del precio de los bienes culturales, se produce una situación de quasimonopsonio donde los productores culturales tienen que adaptarse a las preferencias y presupuestos del Estado (no de los ciudadanos), y donde la garantía de una oferta cultural plural y de calidad depende de la integridad y el buen hacer de quienes dirigen las políticas culturales, pues la capacidad de los ciudadanos para influir en esa oferta y pedir cuentas sobre la misma es muy escasa entre elecciones.

Por el contrario, mediante el mecenazgo ciudadano los ciudadanos recuperan poder para decidir sobre objetivos culturales alternativos mediante sus opciones de inversión y compra. Más poder de influencia en la oferta cultural no es solo poder expresar más preferencias de compra (como en el caso de la soberanía del consumidor de otro tipo de bienes), sino participar mediante el consumo en la vida social. El motivo es que los bienes y servicios culturales tienen contenidos simbólicos relacionados con la auto-comprensión y el conocimiento personal y social. Especialmente el micro-mecenazgo permite una relación más estrecha con los productores culturales, y gracias a la información que las plataformas de crowdfunding proporcionan sobre el desarrollo del proyecto y sobre el comportamiento de los demás micro-mecenas (crowdfunders), el proceso es análogo a una discusión pública sobre la producción de bienes culturales y sobre la definición de los estándares significativos, simbólicos o estéticos que los constituyen (Burtch, Ghose y Wattal, 2013). En suma, soberanía del consumidor cultural se relaciona aquí con empoderamiento, con la función constructiva que la libertad tiene en la determinación de los valores y normas sociales (Sen, 1999), y en consecuencia con el rol de ciudadano. 


\section{REFLEXIONES FINALES}

El análisis conceptual realizado ha expuesto el marco conceptual desde el que abordar la trascendencia ética de los diferentes sistemas de financiación de la Cultura, y por qué los incentivos públicos a los consumidores culturales son una política cultural progresista en el sentido indicado. Aunque existen poderosos argumentos conceptuales en su contra, la financiación de los bienes y servicios culturales mediante un porcentaje significativo de financiación privada también es defendible al margen de restricciones presupuestarias coyunturales. Dejando aparte modas pasajeras sobre las posibilidades del crowdfunding para la financiación cultural, existen argumentos morales genuinos a favor del mecenazgo ciudadano.

Aunque dicho análisis aporta criterios para juzgar comparativamente SFC alternativos, la respuesta final sobre cuál es más recomendable requiere análisis ulteriores con una metodología empírica. Como ocurre en los demás ámbitos de la ética aplicada, los juicios sobre casos concretos dependen de circunstancias empíricas no controlables a priori. Por ejemplo, las decisiones de los micro-mecenas son altamente dependientes de las de los demás participantes en una misma campaña de crowdfunding, lo que puede causar problemas de "comportamiento gregario" (herd behavior) dependiendo del perfil de micro-mecenas predominante (Agrawal, Catalini y Goldfarb, 2015; Burtch, Ghose y Wattal, 2013). Así mismo, la hipótesis del SFC2 requiere para ser razonable una sustancial disminución de las disparidades de poder de compra que ocurren en las sociedades reales. Recordemos que en el SFC2 el presupuesto general para Cultura es igual que si fuera el Estado quien directamente lo gastara. No obstante, como dije en la sección segunda, eso no conlleva automáticamente que cualquier consumidor cultural tenga el mismo poder sobre la oferta, ni por tanto igual capacidad de participar (a través de sus decisiones de consumo e inversión), en el diálogo sobre qué vale la pena en Cultura. El motivo es que la renta disponible para Cultura varía con la renta disponible total, cuya cuantía obviamente es independiente del SFC ele- gido. Además, los individuos podrían preferir fines distintos a la Cultura como destino de los fondos sobre los que el SFC2 les permite decidir. A pesar de todo, los argumentos aportados prueban el potencial crítico y emancipador del mecenazgo ciudadano, a la vez que proporcionan una ilustración de la dimensión ética que tiene el diseño institucional de un sistema de financiación.

Finalmente, el mecenazgo ciudadano ilustra el potencial transformador de las decisiones individuales de consumo e inversión en la sociedad de la información actual. Una respuesta habitual a la injerencia de los Estados en la vida cultural es la sustitución del mecenazgo estatal dirigista por una democracia cultural caracterizada por la descentralización de las políticas culturales (Vidal-Beneyto, 1981). En ese modelo la participación ciudadana es protagonista porque la función primordial del Estado es promover los equipamientos culturales y los canales de difusión que hacen posible la vida cultural de la sociedad. Sin entrar en las críticas del funcionamiento real de este modelo, he propuesto una alternativa distinta al antagonismo de Estado y mercado en el terreno cultural. Se trata de revisar la oposición Estado desinteresado vs. consumidor interesado según la cual, fuera del mecanismo de mercado, solo serían posibles tres categorías: la provisión de bienes públicos por el Estado, la provisión de servicios profesionales a precios regulados por el Estado, y por último donaciones interindividuales en la esfera privada (Anderson, 1993; R. J. G. Claassen, 2009). No se trata de que el mecenazgo cultural sea una categoría más que añadir a ese esquema, sino de que el comportamiento de consumidores e inversores culturales individuales pone en cuestión el esquema mismo. Dicho de otra manera, el mecenazgo ciudadano abre la posibilidad de que el consumo y la inversión individual en bienes culturales sean una forma de participación en la vida social.

\section{AGRADECIMIENTOS}

Investigación parcialmente financiada con cargo al Proyecto I+D+i 2015-2018 Civic Constellation II: Debating Democracy and Rights (FFI2014-52703-P).

\section{NOTAS}

1. Dada la anfibología del término "Cultura", el impacto de las industrias culturales desde la Segunda Guerra Mundial, la emergencia de la economía creativa, e incluso la reflexión estética reciente, definir "Cultura" o "actividad cultural” puede ser largo. Para los propósitos de este artículo basta una definición ostensiva que incluya las actividades calificadas como culturales en la legislación españo- la. En consecuencia, la Cultura incluye (y seguramente no solo): la cinematografía, las artes audiovisuales y las artes multimedia; las artes escénicas, la música, la danza, el teatro y el circo; las artes visuales: artes plásticas o bellas artes, la fotografía y el diseño; las ediciones literarias, fonográficas y cinematográficas, en cualquier soporte o formato; las actividades relacionadas con la investigación, docu-

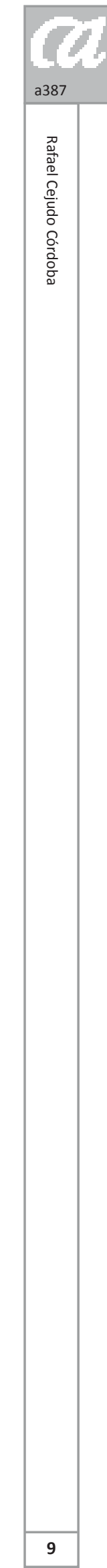

mentación, conservación, restauración, recuperación, difusión y promoción del patrimonio histórico (basándome por ejemplo en el Anteproyecto de ley por el que se adoptan medidas tributarias y administrativas destinadas a estimular la actividad cultural en Andalucía, art. 2b). Solo para evitar la confusión con el sentido etnográfico de "Cultura" escribiré el término con mayúscula. 
2. Se trata de medidas como deducciones por compra de bienes y servicios culturales, deducciones por micro-mecenazgo, por transmisión de empresas culturales, por cesiones de bienes culturales para exhibición, productos financieros de inversión cultural o fomento de empresas públicas de micro-mecenazgo. Medidas de este tipo se han propuesto en el Anteproyecto de ley por el que se adoptan medidas tributarias y administrativas destinadas a estimular la activi-

\section{BIBLIOGRAFÍA}

Adorno, T. W. (1977). Kulturkritik und Gesellschaft. I. Prismen, Ohne Leitbild. Frankfurt am Main: Suhrkamp.

Agrawal, A., Catalini, C. y Goldfarb, A. (2015). Crowdfunding: Geography, Social Networks, and the Timing of Investment Decisions. Journal of Economics and Management Strategy, 24, 2, pp. 253-274. https://doi.org/10.1111/jems.12093

Albi Ibáñez, E. (2003). Economía de las artes y política cultural. Madrid: Instituto de Estudios Fiscales.

Alemany, L. y Bultó, I. (2014). 'Crowdfunding': nueva forma de financiación para los emprendedores. Harvard Deusto Business Review, 237, pp. 6-19.

Anderson, E. S. (1993). Value in Ethics and Economics. Harvard: Harvard University Press.

Anomaly, J. (2015). Public goods and government action. Politics, Philosophy \& Economics, 14, 2, pp. 109-128. https:// doi.org/10.1177/1470594X13505414

Anuario de Estadísticas Culturales 2015. Madrid: Ministerio de Educación, Cultura y Deporte. Disponible en https://www.mecd. gob.es/servicios-al-ciudadano-mecd/dms/ $\mathrm{mecd} /$ servicios-al-ciudadano-mecd/estadisticas/cultura/mc/naec/2015/Anuario_ de_Estadisticas_Culturales_2015.pdf

Arendt, H. (1958). The Human Condition. Chicago: The University of Chicago Press.

Bastien, C., Dubois, V., Freyermuth, A. y Matz, K. (2012). Le politique, l'artiste et le gestionnaire. (Re)configurations locales et (dé)politisation de la culture. Broissieux: Editions du Croquant.

Bauman, Z. (1997). Postmodernity and its Discontents: Polity Press.

Baumol, W. J. y Bowen, W. G. (1966). Performing Arts - The Economic Dilemma. A dad cultural en Andalucía (16.05.2012) el Anteproyecto de Ley de Impulso a la Actividad Cultural y del Mecenazgo (06/06/14) y la Ley Foral 8/2014, de 16 de mayo, reguladora del mecenazgo cultural y de sus incentivos fiscales en la Comunidad Foral de Navarra.

3. La postura de Rawls sobre la neutralidad del Estado en cuestiones culturales es un desiderátum, pero la tesis contraria sobre lo que el Estado debe hacer

Study of Problems Common to Theater, Opera, Music, and Dance. New York: Twentieth Century Fund.

Benkler, Y. (2006). The Wealth of Networks: How Social Production Transforms Markets and Freedom. New Haven: Yale University Press.

Bornstein, D. y Davis, S. (2010). Social Entrepreneurship: What Everyone Needs to Know. New York: Oxford University Press.

Bourdieu, P. (1984). Distinction: A Social Critique of the Judgement of Taste. Cambridge, Mass.: Harvard University Press.

Burtch, G., Ghose, A. y Wattal, S. (2013). An Empirical Examination of the Antecedents and Consequences of Contribution Patterns in Crowd-Funded Markets. Information Systems Research, 24, 3, pp. 499-519. https://doi.org/10.1287/ isre. 1120.0468

Casado da Rocha, A. (2013). Niveles éticos y gestión de residuos: Evaluando el sistema de recogida selectiva "puerta a puerta". Dilemata. Revista Internacional de Éticas Aplicadas, 13, pp. 209-229.

Castells, M. (2000). The Rise of The Network Society: The Information Age: Economy, Society and Culture. New York: Wiley.

Claassen, R. (2011). Public Services on the Market: Issues and Arguments. Public Reason. Journal of Political and Moral Philosophy, 3, 2, pp. 3-12.

Claassen, R. (2013). Public Goods, Mutual Benefits, and Majority Rule. Journal of Social Philosophy, 44, 3, pp. 270-290. https://doi.org/10.1111/josp.12031

Claassen, R. J. G. (2009). Institutional pluralism and the limits of the market. Politics, Philosophy \& Economics, 8, 4, pp. 420-447. https://doi. org/10.1177/1470594X09345479 respecto de la Cultura, y también sobre lo que efectivamente hace, cuenta asimismo con una bibliografía muy abundante (Bourdieu, 1984; Dubois, 1999; Fumaroli, 1991; Sierra Rodríguez y Pereiro Pérez, 2005; Sueue-Shya, 2009; Tortosa, 2009). Quien disienta de un neutralismo à la Rawls tendrá más fácil defender que el Estado sí debe implantar aquel SFC que mejore moralmente a los ciudadanos, o sea, la tesis (b).

Collini, S. (1992). De la financiación pública de la cultura en Gran Bretaña. Debats, 42, pp. 28-34.

Cunnane, K. M. (1999). Maintaining Viewpoint Neutrality for the NEA: National Endowment for the Arts v. Finley. Connecticut Law Review, 31, pp. 1445-1483.

De la Torre Sotoca, J. D. (2013). Tributación de la cultura. Granada: Comares.

Dubois, V. (1999). La politique culturelle. Paris: Belin.

Dworkin, R. (1985). A Matter of Principle. Cambridge, Mass: Harvard University Press.

Echeverría, J. (2008). El Manual de Oslo y la innovación social. Arbor. Ciencia, Pensamiento y Cultura, 184, 732, pp. 609-618.

Feinberg, J. (1994). Not with my tax money. The problem of justifying government subsidies for the arts. Journal of Public Affairs, 8, 2, pp. 101-123.

Fowler, A. (2000). NGDOs as a Moment in History: Beyond Aid to Social Entrepreneurship or Civic Innovation? Third World Quarterly, 21, 4, pp. 637-654. https://doi.org/10.1080/713701063

Fumaroli, M. (1991). L'État culturel: une religion moderne. Paris: Éd. de Fallois.

Galston, W. (1991). Liberal Purposes. Goods, Virtues, and Diversity in the Liberal State. Cambridge: Cambridge Univesity Press. https://doi.org/10.1017/ CB09781139172462

Ginsburgh, V. A. y Throsby, D. (2013). Handbook of the Economics of Art and Culture (vol. II). Oxford: Elsevier Science.

Gutiérrez-Rubi, A. y Freire, J. (2012). Manifiesto Crowd. La empresa y la inteligencia de las multitudes. Barcelona: Laboratorio de Tendencias. 
Horkheimer, M. y Adorno, T. W. (1984). Dialektik der Aufklärung: philosophische Fragmente. Frankfurt am Main: Suhrkamp.

Hutt, W. H. (1990). Economists and the Public: A Study of Competition and Opinion. New Brunswick: Transaction Publishers.

Keat, R. (2000). Cultural Goods and the Limits of the Market. London: Palgrave. https:// doi.org/10.1057/9780230595767

Keat, R. (2011). Political Philosophy and Public Service Broadcasting. Public Reason. Journal of Political and Moral Philosophy, 3, 2, pp. 61-79.

Lawton, K. y Marom, D. (2010). The Crowdfunding Revolution: Social Networking Meets Venture Financing. CreateSpace Independent Publishing Platform.

Libro Verde. Fomentar un marco europeo para la responsabilidad social de las empresas. Bruselas, 2001. Disponible en http://www.igualdadenlaempresa. es/enlaces/webgrafia/docs/fomentarun-marco-europeo-para-la-responsabilidad-social-de-las-empresas-2001.pdf

Mill, J. S. (1977a). Considerations on Representative Government. En Robson, J. M. (ed.). The Collected Works of John Stuart Mill, Vol. XIX - Essays on Politics and Society. Toronto: University of Toronto Press, pp. 371-578.

Mill, J. S. (1977b). Thoughts on Parliamentary Reform 1859. En Robson, J. M. (ed.). The Collected Works of John Stuart Mill, Vol. XIX - Essays on Politics and Society. Toronto: University of Toronto Press, pp. 313-339.

Mulgan, G. (2006). The Process of Social Innovation. Innovations: Technology, Governance, Globalization, 12, 1, pp. 145-162. https://doi.org/10.1162/itgg.2006.1.2.145

Murray, R., Caulier-Grice, J. y Mulgan, G. (2010). The Open Book of Social Innovation. London: The Young Foundation/NESTA. Disponible en https:// youngfoundation.org/wp-content/ uploads/2012/10/The-Open-Book-ofSocial-Innovationg.pdf
Nicholls, A. y Murdock, A. (eds.) (2012). Social Innovation. Blurring Boundaries to Reconfigure Markets. Palgrave Macmillan. https://doi.org/10.1057/9780230367098

Nussbaum, M. (1990). Aristotelian Social Democracy. En Douglass, R. B. (ed.) Liberalism and the Good. New York: Routledge, pp. 203-252.

Observatorio de la Cultura. Primer semestre de 2013. Análisis de resultados. Disponible en http://www.fundacioncontemporanea.com/pdf/ANALISIS_RESULTADOS_OBS_JUNIO_2013.pdf

Persky, J. (1993). Retrospectives: Consumer Sovereignty. Journal of Economic Perspectives, 7, 1, pp. 183-191. https://doi. org/10.1257/jep.7.1.183

Ponce Velasco, P. (2013). Mecenazgo ¿Por qué una reforma normativa? Información Comercial Española, 872, pp. 93-102.

Raussell Köster, P., Montagut Marqués, J. y Minyana Beltran, T. (2013). Hacia nuevos modelos de financiación cultural: ¿renovar el mecenazgo? Periférica, 14, pp. 209-233.

Rawls, J. (1971). A Theory of Justice. Oxford: Oxford University Press.

Raz, J. (1986). The Morality of Freedom. Oxford: Clarendon Press.

Rodríguez Ferrándiz, R. (2011). De industrias culturales a industrias del ocio y creativas: los límites del "campo» cultural. Comunicar: Revista Científica Iberoamericana de Comunicación y Educación, XVIII, 36, pp. 149-156. https://doi. org/10.3916/c36-2011-03-06

Rubio Arostegui, J. A., Rius Ulldemolins, J. y Martínez Illa, S. (2014). El modelo español de financiación de las artes y la cultura en el contexto europeo. Madrid: SGAE/Fundación Alternativas.

Sajardo Moreno, A. y Ribeiro Coutinho, D. (2014). La nueva financiación del bienestar: economía social y crowdfunding. ¿La era de la sociedad mecenas? En: García Linaza, A., Fernández Morales, A. y Podadera Rivera, P. (coords.) Ana- les de economía aplicada, XXVIII. Madrid: Delta Publicaciones Universitarias, pp. 829-845. Disponible en http://www. asepelt.org/ficheros/File/Anales/2014/ anales-2014.pdf

Schumpeter, J. A. (1934). The Theory of Economic Development: An Inquiry Into Profits, Capital, Credit, Interest, and the Business Cycle. Transaction Books.

Sen, A. (1970). Collective Choice and Social Welfare. San Francisco: Holden-Day.

Sen, A. (1999). Development as Freedom. Oxford: Oxford University Press.

Sen, A. (2009). The Idea of Justice. Londres: Penguin.

Sierra Rodríguez, X. C. y Pereiro Pérez, X. (eds.) (2005). Patrimonio cultural: politizaciones y mercantilizaciones. Sevilla: Fundación el Monte. Federación de Asociaciones de Antropología del Estado Español. Asociación Andaluza de Antropología.

Siurana, J. C. (2009). La sociedad ética. Indicadores para evaluar éticamente una sociedad. Barcelona: Proteus.

Sueue-Shya, Z. H. (2009). Andre Malraux's Cultural Policies. Universitas-Monthly Review of Philosophy and Culture, 36, 5, pp. 91-103.

Tortosa, V. (ed.) (2009). Mercado y consumo de ideas: de industria a negocio cultural. Madrid: Biblioteca Nueva.

Vidal-Beneyto, J. (1981). Hacia una fundamentación teórica de la política cultural. REIS, 16, 81, pp. 123-134. https:// doi.org/10.2307/40182969

Vitell, S. J. (2015). A Case for Consumer Social Responsibility (CnSR): Including a Selected Review of Consumer Ethics/ Social Responsibility Research. Journal of Business Ethics, 130, 4, pp. 767-774. https://doi.org/10.1007/s10551-0142110-2 